\title{
Process Development for the Synthesis of Hepatitis C NS5A Protein Inhibitor Odalasvir
}
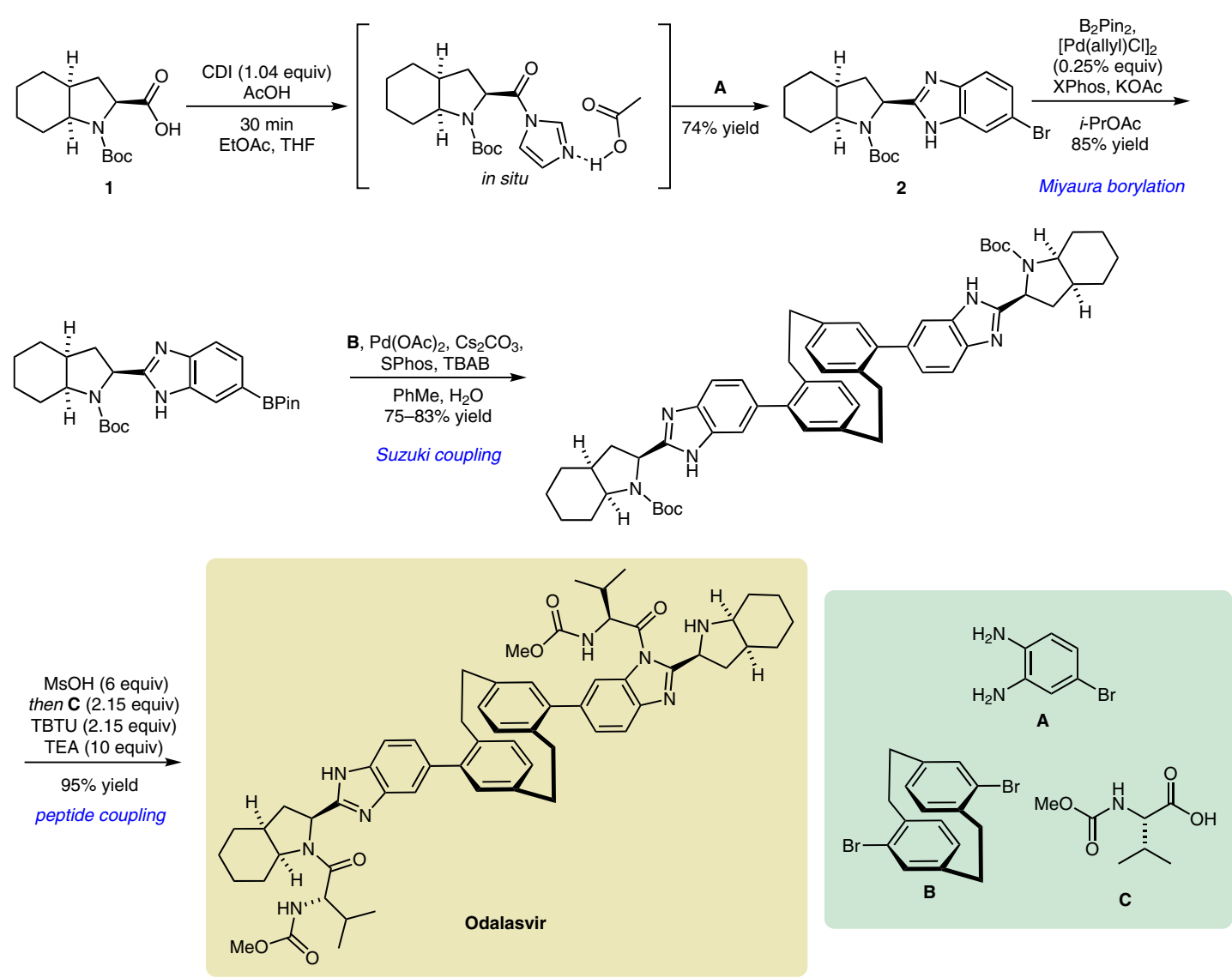

Significance: Chronic hepatitis $C$ infection can be treated by combination therapies. One targeted component is the nonstructural protein 5A (NS5A) that is involved in viral replication. NS5A inhibitors are usually dimeric in nature. Odalasvir is a NS5A inhibitor, where two benzimidazole-octahydroindole ligands are linked by a paracyclophane unit. Here, the authors describe the process development for Odalasvir, where the process mass intensity (PMI) was improved, catalyst loadings minimized, and steps telescoped to yield the ligand for the Phase 3 clinical stage.
Comment: Octahydroindole carboxylic acid 1 was treated with CDI to effect full conversion to the acyl imidazole, which, upon activation with $\mathrm{AcOH}$, was amidated with aniline $\mathbf{A}$. AcOH further promoted the cyclization to benzimidazole 2 . The catalyst loading of $[\mathrm{Pd}(\mathrm{allyl}) \mathrm{Cl}]_{2}$ for aryl bromide borylation was reduced to $0.25 \mathrm{~mol} \%$, and the solvent $i$-PrOAc allowed phase separation during workup. The paracyclophane linker was introduced by double Suzuki coupling with B. Finally, double Boc deprotection was mediated by $\mathrm{MsOH}$, followed by double peptide coupling to L-Moc-Val (C) to yield Odalasvir. The process can be scaled to $100 \mathrm{~kg}$ batches.
Key words

large-scale synthesis

paracyclophane

hepatitis C

antiviral agents 